\title{
Transceiver Design and Power Allocation for SWIPT in MIMO Cognitive Radio Systems
}

\author{
Fahui $\mathrm{Wu}^{1}{ }^{1}$, Lin Xiao ${ }^{2, *}$, Dingcheng Yang ${ }^{2}$, Laurie Cuthbert ${ }^{3}$ and Xiaoping Liu ${ }^{1,4}$ \\ 1 Jiangxi Province Key Laboratory of Intelligent Information Systems, Nanchang University, \\ Nanchang 330031, China; fahui97@email.ncu.edu.cn (F.W.); xpliu@sce.carleton.ca (X.L.) \\ 2 Information Engineering School, Nanchang University, Nanchang 330031, China; \\ yangdingcheng@ncu.edu.cn \\ 3 Information Systems Research Centre, Macao Polytechnic Institute, Macao 999078, China; \\ laurie.cuthbert@isrcmo.org \\ 4 Department of Systems and Computer Engineering, Carleton University, Ottawa, ON K1S 5B6, Canada \\ * Correspondence: xiaolin@ncu.edu.cn
}

Received: 17 October 2018; Accepted: 12 November 2018; Published: 18 November 2018

\begin{abstract}
In this paper, we consider a symmetric wireless communication network, i.e., each user is equipped with the same number of antennas. Specifically, this paper studies simultaneous wireless information and power transfer (SWIPT) in a K-user multiple-input multiple-output (MIMO) cognitive radio network where the secondary users (SUs) access the same frequency band as the pre-existing primary user (PU) without generating any interference. The transceivers and power splitting ratio are designed and power allocation is considered in our system model. To guarantee the signal-to-interference-plus-noise ratio (SINR) and harvested energy requirement of the PU, its optimal transceiver and minimal transmitted power are obtained by the technique of semi-definite relaxation (SDR). We design the beamformers of the SUs using the distance between the interference subspaces at the PU and the null space of PU's desired signal to preserve the PU from the interference caused by the SUs. We aim to maximize the sum rate of all the SUs by jointly designing power splitting ratios and allocating transmission power. Furthermore, to consider the performance fairness of SUs, we propose another approach to maximize the minimum SINR of the SUs. Finally, numerical results are given to evaluate the performance of proposed approaches.
\end{abstract}

Keywords: simultaneous wireless information and power transfer (SWIPT); power splitting; cognitive radio; multiple-input multiple-output (MIMO)

\section{Introduction}

Wireless power transfer (WPT) techniques have generated interest from both academia and industry over the past decades [1-7]. Generally speaking, WPT can be achieved via mainly three methods, i.e., inductive coupling [1,2], magnetic resonant coupling [3,4] for near-field WPT, and electromagnetic (EM) radiation $[5,6]$ is a far-field WPT technology, also known as radiative WPT. In radiative WPT, users convert the received radio frequency (RF) signal into electricity. Compared to the two near-field WPT techniques, radiative WPT can implement power transfer over distances varying from a few meters to several kilometers. As a result, radiative WPT is considered as a suitable solution to support next-generation wireless communication networks without the need to charge batteries via electrical wire. This is crucial for those devices which cannot be charged via wire, such as sensors embedded in body or building. Since RF signals can carry energy as well as information, combining RF wireless power transfer and information transmission leads to the simultaneous wireless information and power transfer (SWIPT) [6]. This concept was proposed firstly 
in [8], where the authors characterized the rate-energy regions for point-to-point SWPIT systems with noisy channels. In [6], a three-user multiple-input multiple-output (MIMO) broadcast systems for SWIPT was investigated, with two practical receiver structures to enable SWIPT being proposed: (i) "time switching" (TS) with the receiver switching its operations periodically between energy harvesting (EH) and information decoding (ID); and (ii) "power splitting" (PS) with the receiver splitting the received signal into two streams, one for EH and another for ID. With the practical SWIPT schemes introduced above, the applications of SWIPT have been investigated in various network configurations, such as point-to-point single-antenna channel [9], orthogonal frequency division multiplexing (OFDM)-based multiple users system [10], and relay channel [11].

\subsection{Related Works}

One of the key challenges in most wireless communication systems is interference, and there is no exception in multi-user SWIPT systems. In this paper, we pay attention to performing SWIPT in a cognitive interference channel (IFC), in which there is one primary transmitter-receiver pair and several secondary user pairs. According to the methods applied for interference management, prior research on SWIPT in IFC network can be loosely classified into two categories. In the first category, the beamforming matrix and the PS ratio are jointly optimized [12-15]. In [12], a multiple-input single-output (MISO) systems with perfect channel statement information (CSI) is investigated for SWIPT, and a centralized algorithm is proposed to solve joint beamforming and power splitting (JBPS) problem. Then, this work is extended to imperfect CSI at the transmitters in [13], the proposed decentralized algorithm being guaranteed to coverage to the centralized solution. In a more complex K-pair MIMO interference channel [14], three transceiver designs with different computational complexity are proposed to solve the JBPS problem. However, an oversimple, identical PS ratio over all the receiver antennas is assumed in [14]. To achieve a more optimal PS scheme, with a full-duplex K-pair MIMO interference channel scenario [15], the authors took into account a PS vector (i.e., different ratios for different receive antennas). The main objective of those works is to minimize the total transmit power, while satisfying the signal-to-interference-plus-noise ratio (SINR) and harvested energy constraints (i.e., IT and EH requirements guaranteed). Due to the nonconvexity of the studied JBPS problem, the intended problem can usually be relaxed as a tractable (centralized) semidefinite program or second-order cone program, thus the developed solutions have lower computational complexity but suboptimal performance.

Another category considers the interference alignment (IA) to manage the interference in EH IFC [16-19]. In IA-based networks, each user's precoder can be cooperatively designed such that the interference at all the receivers only occupies a small subspace, so the intended information can be reconstructed without interference through the decoder [16]. When applying IA to SWIPT networks, we can assume the signal stream fed into the ID receiver to be free of interference, thus bringing great convenience [17]. In [17], the authors first studied the joint transmit power allocation and power splitting for IA, and IA was implemented via antenna selection in [18]. Moreover, the security issue in IA based SWIPT was also investigated in [19]. As a matter of fact, there is less research on the IA based SWIPT.

On the other hand, since cognitive radio (CR) has been considered as a promising technique to solve the spectrum scarcity problem, many spectrum sensing schemes are developed for $\mathrm{CR}$ networks [20-23]; the CR SWIPT in CR networks also has attracted much attention [24-29]. The outage performance of SWIPT is studied in [24] for a cognitive relay network. For MISO cognitive radio systems, authors in [25] investigated the physical-layer secrecy problem, to protect the secrecy rate and energy harvesting of the primary user (PU), with a robust artificial noise aided beamforming and power splitting design being proposed under imperfect CSI. In the same system, authors in [26] jointly optimized beamforming and found power splitting ratios for the Max-Min problem by using semidefinite relaxation (SDR). In an underlay model MIMO CR network [27], a robust transceiver is designed for SWIPT, wherein the authors modeled the channel uncertainties by the worst-case model; 
they maximized the sum of harvested power at all the EH receivers under the minimum information rate constraints at the secondary user (SU) and interference constraint at the PU. The physical layer secrecy problem is studied in a CR MIMO network in [28]; the authors investigated the artificial noise (AN)-aided precoding design problem and formulated the problem as a secrecy rate maximization problem, this problem being solved by a successive convex approximation method. Furthermore, the outage-constrained secrecy rate maximization problem is analyzed in [29], the transmit covariance matrix and artificial noise covariance matrix being optimized to maximize the secrecy rate.

\subsection{Motivation and Contributions}

In a CR network, specifically, an underlay spectrum sharing CR network, the power of interference and noise at the primary receiver is constrained by the interference temperature limit (ITL), so that it is important to carefully control transmit power and to design the transmission precoder for SUs to successfully share spectrum. However, the interference from SUs can be salvaged as energy if the PU receiver is a SWIPT user. From the point of view of a SWIPT PU, maximizing the harvested $\mathrm{RF}$ energy and minimizing the interference level are contradictory. Fortunately, the precoder design scheme of interference alignment IA can eliminate interference perfectly. When IA is applied to SWIPT, the interference-free signal steams are fed to the ID receiver, and the interference is fed to the EH receiver for energy harvesting. Hence, in this paper, the interference from SUs to the PU is aligned into a subspace in the PU. After that, to further improve the performance of the system, we consider the power allocation (PA) among all users. To the best of our knowledge, there is no other existing work accounting for the JBPS and power allocation of SWIPT in MIMO CR networks. The distinct features of our paper are as follows:

- The transceiver design and power allocation problem in a MIMO CR network is studied, and an interference-alignment-based precoder design scheme for the SUs is proposed to protect the priority of the PU. This problem is solved by alternating optimization and convex optimization. The minimum transmit power, optimal transceiver and power splitting ratio of the PU are derived to guarantee its SINR and harvested energy constraints by using the SDR technique.

- The precoder of the SUs is analyzed by the theory of minimum squared Euclidean distance. The precoder of the SUs is obtained by eigenvalue decomposition of the interference covariance matrix.

- We propose a PA algorithm to maximize the sum rate of SUs. As the sum rate maximization power allocation algorithm may compromise some user's performance, we further propose a PA algorithm to maximize the minimum SINR of the SUs.

- The approaches proposed can be implemented in the CR network especially the unlicensed spectrum CR where a PU's interest must be protected. Moreover, our solutions can be extended to traditional communication network without WPT.

\subsection{Organization and Notation}

The rest of this paper is organized as follows. Section 2 presents the system model and problem formulation. The sum rate maximization solution and Max-Min solution are presented in Section 3. Section 4 presents numerical simulation results and analysis to validate our solutions. Section 5 concludes the paper.

In this paper, we denote the scalars by lower-case letters, bold-face lower-case letter are used for vectors, $\mathbf{A}$ is a matrix, and $\mathcal{A}$ is a set. For a square matrix $\mathbf{A}, \operatorname{Rank}(\mathbf{A}), \operatorname{Tr}(\mathbf{A}), \mathbf{A}^{T}$, and $\mathbf{A}^{H}$ denote its trace, rank, transpose and conjugate transpose, respectively. $\|\cdot\|$ denotes the Euclidean norm of a vector, $|\cdot|$ denotes the absolute value a scalar, and $\mathbf{A} \succeq \mathbf{0}$ means $\mathbf{A}$ is a Hermitian positive semi-definite matrix. $\mathbf{I}_{m}$ denotes an $m$ by $m$ identity matrix, and $\mathbb{C}^{m \times n}$ denotes the space of $m \times n$ complex matrices. The distribution of a circularly symmetric complex Gaussian (CSCG) random vector with mean $\mu$ and covariance matrix $\mathbf{C}$ is represented as $\mathcal{C N}(\mu, \mathbf{C})$. 


\section{System Model and Problem Formulation}

\subsection{System Model}

In this section, we present the system model and the formulation of our problem. As shown in Figure 1, consider a K-user interference CR network that consists of a single primary link and $K-1$ secondary transmissions. The secondary users are allowed to share the licensed spectrum with primary user as long as the interference temperature limit of the PU can be ensured. All the transmitters simultaneously transfer information and energy to corresponding receivers, i.e., $K$-pair SWIPT users. Each transmitter and receiver is equipped with $M_{k}$ and $N_{k}$ antennas, respectively. The $k$ th user transmits uncorrelated symbols $x_{k}$ which consist of $d_{k} \leq \min \left\{M_{k}, N_{k}\right\}$ data streams with transmitting power $P_{k}$, i.e., $\mathbb{E}\left[\left\|\mathbf{x}_{k}\right\|^{2}\right]=P_{k}, \mathbf{x}_{k} \sim \mathcal{C N}(0,1)$.

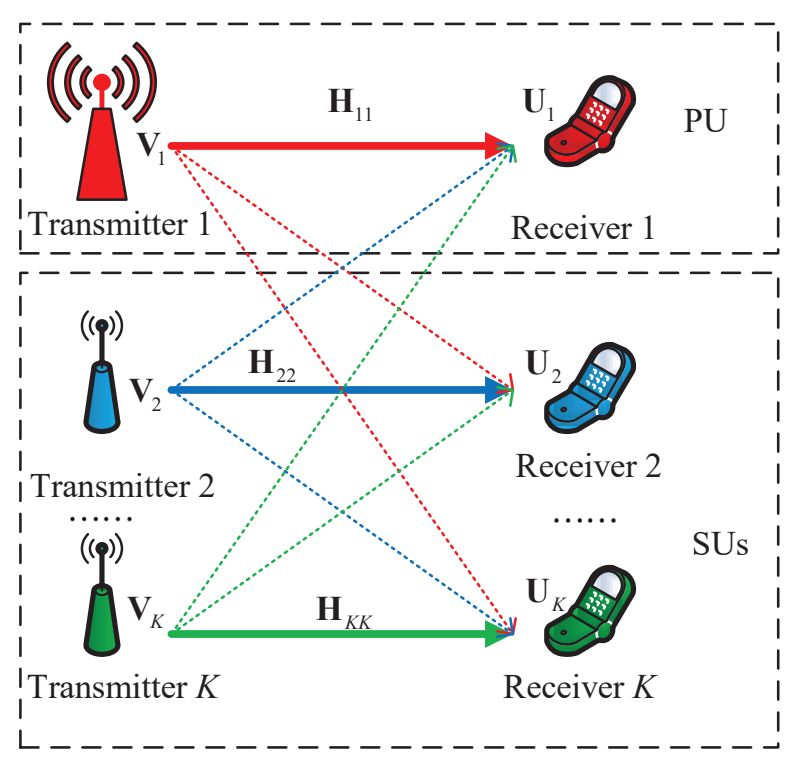

Figure 1. System model.

To manage and eliminate interference and thus get a higher transmission degree of freedom (DoF), IA can be adopted on the network, which is called "IA-based CR network". IA tries to jointly design the precoder matrix for all transmitters so the that the desired signal can be extracted by a decoding matrix. Let $\mathbf{V}_{k}$ of dimension $M_{k} \times d_{k}$ and $\mathbf{U}_{k}$ of dimension $N_{k} \times d_{k}$, respectively, denote the precoding matrix of the $k$ th transmitter and decoding matrix of $k$ th receiver. The received signal at the $k$ th user with perfect synchronization is:

$$
\mathbf{y}_{k}=\mathbf{U}_{k}^{H} \mathbf{H}_{k k} \mathbf{V}_{k} \mathbf{x}_{k}+\sum_{i=1 . i \neq k}^{K} \mathbf{U}_{k}^{H} \mathbf{H}_{k i} \mathbf{V}_{i} \mathbf{x}_{i}+\mathbf{U}_{k}^{H} \mathbf{z}_{k}
$$

where $\mathbf{H}_{k i}$ is the channel coefficient matrix from the $i$ th transmitter to the $k$ th receiver. $\mathbf{z}_{k}$ represents the additive white Gaussian noise vector (AWGN) at the $k$ th receiver with distribution $\mathcal{C N}\left(\mathbf{0}, \sigma^{2} \mathbf{I}_{N_{k}}\right)$. When IA is performed, the received interference at the $k$ th receiver is constrained into the same subspace that is orthogonal to the interference suppression matrix $\mathbf{U}_{k}$. Thus, the desired signal can be recovered from the remaining interference-free subspace. More specifically, when IA is feasible, the following conditions need to be satisfied:

$$
\begin{array}{r}
\mathbf{U}_{k}^{H} \mathbf{H}_{k j} \mathbf{V}_{j}=\mathbf{0}, \forall j \neq k \\
\operatorname{Rank}\left(\mathbf{U}_{k}^{H} \mathbf{H}_{k k} \mathbf{V}_{k}\right)=d_{k}
\end{array}
$$


Similar to [30], this paper considers that all users have the same transceiver antennas and data streams, i.e., $M_{k}=M, N_{k}=N$, and $d_{k}=d$, thus the feasible condition for IA in this paper can be written as

$$
d \leq \frac{N}{2 K+1}+\frac{K M}{2 K+1}
$$

We assume that this condition holds throughout this paper.

In this paper, we assume each receiver is equipped with a power splitter to process the received signal. The received signal is split into two separate signal streams with different power levels, one sent to the ID receiver and the remaining fed to the EH receiver. Thus, the signal split to the the ID of $k$ th receiver with perfect interference elimination is expressed as:

$$
\mathbf{y}_{k}^{\mathrm{ID}}=\sqrt{\rho_{k}}\left(\mathbf{U}_{k}^{H} \mathbf{H}_{k k} \mathbf{V}_{k} \mathbf{x}_{k}+\mathbf{z}_{k}\right)+\mathbf{n}_{k}
$$

where $\mathbf{n}_{k} \sim \mathcal{C N}\left(0, \delta^{2} \mathbf{I}_{N_{k}}\right)$ is the additional processing noise introduced when operate ID at receiver $K$. Accordingly, the SINR of the $k$ th user with perfect IA can be calculated as:

$$
\operatorname{SINR}_{k}=P_{k} \frac{\rho_{k}}{\rho_{k} \sigma^{2}+\delta^{2}} \mathbf{U}_{k}^{H} \mathbf{H}_{k k} \mathbf{V}_{k} \mathbf{V}_{k}^{H} \mathbf{H}_{k k}^{H} \mathbf{U}_{k}
$$

On the other hand, the signal split to the $\mathrm{EH}$ of the $k$ th receiver is given by:

$$
\mathbf{y}_{k}^{\mathrm{EH}}=\sqrt{1-\rho_{k}}\left(\sum_{i=1}^{K} \mathbf{H}_{k i} \mathbf{V}_{i} \mathbf{x}_{i}+\mathbf{z}_{k}\right)
$$

Then, the energy harvested by the $\mathrm{EH}$ of the $k$ th user is given by

$$
E_{k}=\zeta_{k}\left(1-\rho_{k}\right)\left(\sum_{i=1}^{K} P_{k}\left\|\mathbf{H}_{k i} \mathbf{V}_{i}\right\|^{2}+\sigma_{k}^{2}\right)
$$

where $\zeta_{k}\left(0<\zeta_{k} \leq 1\right)$ is the energy conversion efficiency at the EH of $k$ th receiver. When IA is leveraged in the SWIPT based CR network, not only can ID receive an interference-free signal, but it can also re-utilize the interference as an energy source rather discard it. Therefore, IA can provide an efficient interference management solution for the SWIPT based CR network, in which the interference becomes a benefit and so does not need be discarded.

However, with IA, the SINR of the receiver may decrease in moderate or low SNR situations; this problem is something that should be avoided since the performance of the PU must not be degraded, as that is a key objective of a CR network. To this end, we consider a situation where the PU neglects the presence of the SUs and retains their own transmission power and transmission beamforming. The SUs should design their preprocessing matrices and control their transmission power to guarantee the PU's QoS, otherwise the SUs will not be allowed to access the licensed spectrum. Hence, the interference from SUs is aligned at PU, and the SINR of PU is expressed as

$$
\operatorname{SINR}_{1}=P_{1} \frac{\rho_{1}}{\rho_{1} \sigma_{1}^{2}+\delta_{1}^{2}} \mathbf{U}_{1}^{H} \mathbf{H}_{11} \mathbf{V}_{1} \mathbf{V}_{1}^{H} \mathbf{H}_{11}^{H} \mathbf{U}_{1}
$$

Without loss of generality, to ensure the PU's SINR metrics independent of the power of transmit/receive filters, we assume $\left\|\mathbf{U}_{1}\right\|=1$ and $\left\|\mathbf{V}_{1}\right\|=1$ in the following. As an SU will always suffer the interference from PU and other SUs, then the SINR of SUs is expressed as

$$
\operatorname{SINR}_{k}=\frac{P_{k} \rho_{k}\left|\mathbf{U}_{k}^{H} \mathbf{H}_{k k} \mathbf{V}_{k}\right|^{2}}{\sum_{j \neq k}^{K} \rho_{k}\left|\mathbf{U}_{k}^{H} \mathbf{H}_{k j} \mathbf{V}_{j}\right|^{2}+\rho_{k} \sigma_{k}^{2}\left\|\mathbf{U}_{k}\right\|^{2}+\delta_{k}^{2}\left\|\mathbf{U}_{k}\right\|^{2}}
$$


where $k \in \hat{\mathcal{K}}$, the set $\hat{\mathcal{K}}=\{2,3, \ldots, K\}$. Similarly, $\left\|\mathbf{U}_{k}\right\|=1, k \in \hat{\mathcal{K}}$ is assumed. It should be noted that, in this paper, it is assumed that perfect knowledge of the channel is available at the PU, and the SUs have perfect knowledge of the all channel matrices (perfect CSI of the primary link is also available at the SUs). The power allocation is considered for sum rate maximization among SUs, and their postprocessing matrices are designed to mitigate the received interference from the PU to improve their own performances. We consider the joint power allocation and pre/postprocessing matrices design for the CR-SWIPT network under these assumptions.

\subsection{Problem Formulation}

Considering that the only objective of the PU's precoding matrix design is to satisfy the QoS requirement on both SINR and harvested energy, interference from the PU to SUs may be ignored. The interference from the SUs to the PU is concentrated by the SU's precoding matrices, and then eliminated by the PU's decoding matrix. Moreover, a minimum harvested energy in the SUs should be maintained to accommodate the receiver-side basic functions. Under these assumptions, we aim to maximize the sum rate of all SUs. The joint power allocation and transceiver design problem can be formulated as:

$$
\begin{array}{ll}
\max _{\left\{\mathbf{V}_{k}, \mathbf{U}_{k}, \rho_{k}, P_{k}\right\}} \sum_{k=2}^{K} \operatorname{SINR}_{k}, \\
\text { s.t. } & P_{1} \frac{\rho_{1}}{\rho_{1} \sigma_{1}^{2}+\delta_{1}^{2}} \mathbf{U}_{1}^{H} \mathbf{H}_{11} \mathbf{V}_{1} \mathbf{V}_{1}^{H} \mathbf{H}_{11}^{H} \mathbf{U}_{1} \geq \gamma_{1}, \\
& \zeta_{k}\left(1-\rho_{k}\right)\left(\sum_{j=1}^{K} P_{j}\left\|\mathbf{H}_{j k} \mathbf{V}_{j}\right\|^{2}+\sigma_{k}^{2}\right) \geq e_{k}, k \in \mathcal{K}, \\
& 0 \leq \rho_{k} \leq 1, k \in \mathcal{K}, \\
& \left\|\mathbf{V}_{k}\right\|^{2}=1, k \in \mathcal{K}, \\
& \mathbf{U}_{1}^{H} \mathbf{H}_{1 k} \mathbf{V}_{k}=\mathbf{0}, k \in \hat{\mathcal{K}}, \\
& \mathbf{V}_{k}^{H} \mathbf{V}_{k}=\mathbf{I}, \mathbf{U}_{k}^{H} \mathbf{U}_{k}=\mathbf{I}, k \in \hat{\mathcal{K}}, \\
& \left\|\mathbf{U}_{1}\right\|^{2}=1,\left\|\mathbf{V}_{k}\right\|^{2}=1, \sum_{k=1}^{K} P_{k}=P_{\text {total }} .
\end{array}
$$

It is assumed that $\gamma_{1}>0$ and all receivers have non-zero harvested energy targets, i.e., $e_{k}>0, \forall k$; thus, the received PS ratios at all receivers should satisfy $0<\rho_{k}<1, \forall k$. We can observe that the problem is nonconvex because the variables $\left\{\mathbf{V}_{k}\right\},\left\{\mathbf{U}_{k}\right\},\left\{\rho_{k}\right\}$, and $\left\{P_{k}\right\}$ are coupled together in SINR constraints and EH constraints. This problem is still nonconvex even if fixing values of $\rho_{k}$ with $0<\rho_{k}<1, \forall k$. The problem in Equation (9) is nonconvex and finding its global optimum is NP-hard. In the following, we propose an alternating optimization based solution to iteratively solve the problem.

\section{Alternating Optimization}

\subsection{Transceiver Design and Power Allocation for PU}

Note that the PU self-centered performs its energy and information transmission; when PA among users is considered, the SINR and energy harvesting constraints of the PU should be satisfied. We first derive the minimum transmitted power $P_{1}^{*}$, optimal transceiver, i.e., $\mathbf{V}_{1}^{*}, \mathbf{U}_{1}^{*}$, and optimal power splitting ratio $\rho_{1}^{*}$ of the PU that can guarantee its transmission threshold $\gamma_{1}$ and $e_{1}$. This problem can be expressed as follows: 


$$
\begin{array}{ll}
\min _{\mathbf{V}_{1}, \mathbf{U}_{1}, \rho_{1},\left\{P_{k}\right\}} P_{1} \\
\text { s.t. } & P_{1} \frac{\rho_{1}}{\rho_{1} \sigma_{1}^{2}+\delta_{1}^{2}} \mathbf{U}_{1}^{H} \mathbf{H}_{11} \mathbf{V}_{1} \mathbf{V}_{1}^{H} \mathbf{H}_{11}^{H} \mathbf{U}_{1} \geq \gamma_{1}, \\
& \zeta_{1}\left(1-\rho_{1}\right)\left(\sum_{k=1}^{K} P_{k}\left\|\mathbf{H}_{1 k} \mathbf{V}_{k}\right\|^{2}+\sigma_{1}^{2}\right) \geq e_{1}, \\
& 0 \leq \rho_{1} \leq 1, \\
& \left\|\mathbf{U}_{1}\right\|^{2}=1,\left\|\mathbf{V}_{1}\right\|^{2}=1, \sum_{k=1}^{K} P_{k}=P_{\text {total }} .
\end{array}
$$

For Equation (10), we have two observations. First, the SINR of the PU is immune to the SUs' transmissions because of the IA; the PU's decoder $\mathbf{U}_{1}$ is selfishly designed to improve its SINR. The closed form of optimal $\mathbf{U}_{1}^{*}$ that maximizes the $\operatorname{SINR}_{1}$ is [16]:

$$
\mathbf{U}_{1}^{*}=\mathbf{H}_{11} \mathbf{V}_{1}^{*} /\left\|\mathbf{H}_{11} \mathbf{V}_{1}^{*}\right\|
$$

Second, the presence of SUs provides additional energy resource, i.e., interference to the PU's EH receiver; note that $\mathbf{V}_{k}^{H} \mathbf{V}_{k}=\mathbf{I}, k \in \hat{\mathcal{K}}$, hence harvested energy at the PU turns into $Q_{1}=\zeta_{1}\left(1-\rho_{1}\right)\left(P_{1}\left\|\mathbf{H}_{11} \mathbf{V}_{1}\right\|^{2}+\sum_{k=2}^{K} P_{k}\left\|\mathbf{H}_{1 k}\right\|^{2}\right)$. Let $Q_{\text {sUs-PU }}=\sum_{k=2}^{K} P_{k}\left\|\mathbf{H}_{1 k}\right\|^{2}$ denote the harvested energy portion that comes from SUs. If we fix the value of variables $Q_{S U s-P U}$ and $\mathbf{U}_{1}$, and satisfy $\left\|\mathbf{U}_{1}\right\|^{2}=1$ (the detail to choose QSUs-PU and $\mathbf{U}_{1}$ can be seen in the end of Section 3.2), the problem becomes:

$$
\begin{aligned}
& \min _{\mathbf{V}_{1}, \rho_{1}, P_{1}} P_{1} \\
\text { s.t. } & P_{1} \frac{\rho_{1}}{\rho_{1} \sigma_{1}^{2}+\delta_{1}^{2}} \mathbf{U}_{1}^{H} \mathbf{H}_{11} \mathbf{V}_{1} \mathbf{V}_{1}^{H} \mathbf{H}_{11}^{H} \mathbf{U}_{1} \geq \gamma_{1}, \\
& \zeta_{1}\left(1-\rho_{1}\right)\left(P_{1}\left\|\mathbf{H}_{11} \mathbf{V}_{1}\right\|^{2}+Q_{\mathrm{SUs}-\mathrm{PU}}\right) \geq e_{1}, \\
& 0 \leq P_{1} \leq P_{\max },\left\|\mathbf{V}_{1}\right\|^{2}=1,0 \leq \rho_{1} \leq 1 .
\end{aligned}
$$

The problem in Equation (12) is still nonconvex. Let $\mathbf{X}_{1}=P_{1} \mathbf{V}_{1} \mathbf{V}_{1}^{H}$, and then $\operatorname{Rank}\left(\mathbf{X}_{1}\right) \leq 1$. By discarding the rank-one constraint, the SDR of problem with reformulating is given by

$$
\begin{array}{ll}
\min _{\mathbf{X}_{1}, \rho_{1}} \operatorname{Tr}\left(\mathbf{X}_{1}\right) & \\
\text { s.t. } & \frac{1}{\gamma_{1}} \mathbf{U}_{1}^{H} \mathbf{H}_{11} \mathbf{X}_{1} \mathbf{H}_{11}^{H} \mathbf{U}_{1} \geq \sigma_{1}^{2}+\frac{\delta_{1}^{2}}{\rho_{1}}, \\
& \left(Q_{\text {SUs-PU }}+\left\|\mathbf{H}_{11} \mathbf{X}_{1} \mathbf{H}_{11}^{H}\right\|^{2}+\sigma_{1}^{2}\right) \geq \frac{e_{1}}{\zeta_{1}\left(1-\rho_{1}\right)}, \\
& \mathbf{X}_{1} \succeq \mathbf{0}, \quad 0 \leq \rho_{1} \leq 1 .
\end{array}
$$

Note that the SDR problem is convex, as both $1 / \rho_{1}$ and $1 /\left(1-\rho_{1}\right)$ are convex functions over the convex set $0<\rho_{1}<1$, so it can be solved using some existing convex optimization solvers, e.g., CVX [31]. Define $\mathbf{X}_{1}^{*}$ and $\rho_{1}^{*}$ as the optimal solution to the problem; if $\mathbf{X}_{1}^{*}$ is indeed rank-one, then the optimal precoder can be obtained from the eigenvalue decomposition (EVD) of $\mathbf{X}_{1}^{*}$ expressed by $\mathbf{X}_{1}^{*}=\mathbf{x}_{1} \lambda \mathbf{x}_{1}^{H}$, where $\lambda$ denotes the only eigenvalue of $\mathbf{X}_{1}^{*}$, hence the optimal precoder of PU is $\mathbf{V}_{1}^{*}=\mathbf{x}_{1}$ and the optimal transmission power is $P_{1}^{*}=\lambda$ as $\operatorname{Tr}\left(\mathbf{X}_{1}^{*}\right)=\lambda$. Our only concern is whether the optimal solution $\mathbf{X}_{1}^{*}$ satisfies the rank-one constraint, but, fortunately, it can be proved that the solution satisfies $\operatorname{Rank}\left(\mathbf{X}_{1}\right)=1$ by following proposition. 
Proposition 1. The optimal solution $\mathbf{X}_{1}^{*}$ and $\rho_{1}^{*}$ of the problem with given $\gamma_{1}>0$ and $e_{1}>0$, leads to:

1. $\quad \mathbf{X}_{1}^{*}$ satisfies $\operatorname{Rank}\left(\mathbf{X}_{1}^{*}\right)=1$.

2. $\mathbf{X}_{1}^{*}$ and $\rho_{1}^{*}$ satisfy SINR and EH constraint of the problem in Equation (14) with equality.

Proof of Proposition 1. This proposition can be proved by duality theory since the problem in Equation (14) is convex and satisfies Slater's condition (discussed in more detail in Appendix A). The proposition indicates that the rank relaxation on $\mathbf{X}_{1}$ brings no loss of optimality to the problem, i.e., the SDR is tight.

\subsection{Transceiver Design and Power Allocation for SUs}

Next, we design the transceiver $\mathbf{V}_{k}, \mathbf{U}_{k}$, and optimize the power allocation $P_{k}$ and the power splitter $\rho_{k}$ for the secondary links to maximize their sum rate; the optimization problem is formulated as:

$$
\begin{aligned}
& \max _{\left\{\mathbf{V}_{k}, \mathbf{U}_{k}, \rho_{k}, P_{k}\right\}} \sum_{k=2}^{K} \operatorname{SINR}_{k}, \\
& \text { s.t. } \zeta_{k}\left(1-\rho_{k}\right)\left(\sum_{j=1}^{K} P_{j}\left\|\mathbf{H}_{j k} \mathbf{V}_{j}\right\|^{2}+\sigma_{k}^{2}\right) \geq e_{k}, \\
& \quad \sum_{k=2}^{K} P_{k}\left\|\mathbf{H}_{1 k}\right\|^{2} \geq Q_{\text {SUs-PU, }} \\
& \quad \mathbf{U}_{1}^{H} \mathbf{H}_{1 k} \mathbf{V}_{k}=\mathbf{0}, \mathbf{V}_{k}^{H} \mathbf{V}_{k}=\mathbf{I},\left\|\mathbf{U}_{k}\right\|^{2}=1, \\
& \quad 0 \leq \rho_{k} \leq 1, \sum_{k=2}^{K} P_{k}=P_{\text {total }}-P_{1}^{*} ; k \in \hat{\mathcal{K}} .
\end{aligned}
$$

It can be easily verified that the problem is nonconvex, thus the problem is difficult to be directly solved. In the following, we consider a suboptimal algorithm to iteratively design transceiver $\mathbf{V}_{k}$, $\mathbf{U}_{k}$ with fixed $P_{k}$ and $\rho_{k}$, or optimize $P_{k}, \rho_{k}$ with given $\mathbf{V}_{k}, \mathbf{U}_{k}$. The $\mathbf{V}_{k}, \mathbf{U}_{k}$ with given $\rho_{k}$ and $P_{k}$ is optimized as:

$$
\begin{gathered}
\max _{\left\{\mathbf{V}_{k}, \mathbf{U}_{k}\right\}} \sum_{k=2}^{K} \operatorname{SINR}_{k}, \\
\text { s.t. } \mathbf{U}_{1}^{H} \mathbf{H}_{1 k} \mathbf{V}_{k}=\mathbf{0}, \\
\mathbf{V}_{k}^{H} \mathbf{V}_{k}=\mathbf{I}, \\
\left\|\mathbf{U}_{k}\right\|^{2}=1 ; k \in \hat{\mathcal{K}} .
\end{gathered}
$$

The first constraints in Equation (16) indicate that SUs can transmit their signals without causing any interference to the PU. These constraints can be rewritten as follows:

$$
\overline{\mathbf{H}}_{k} \mathbf{V}_{k}=\mathbf{0}, \overline{\mathbf{H}}_{k} \triangleq \mathbf{U}_{1}^{H} \mathbf{H}_{1 k}, \quad k \in \hat{\mathcal{K}}
$$

Equation (17) indicates that the subspace spanned by the matrix $\overline{\mathbf{H}}_{k}$ to be orthogonal the subspace which spanned by the columns of the transmitter of each SU $\left(\mathbf{V}_{k}, k=2,3, \ldots, K\right)$. Furthermore, to convert the IA problem with cognitive constraints into a standard IA problem, we have the following definitions:

$$
\mathbf{V}_{k} \triangleq \mathbf{A}_{k} \tilde{\mathbf{V}}_{k}, k \in \hat{\mathcal{K}}
$$

where $\mathbf{A}_{k}$ and $\tilde{\mathbf{V}}_{k}$ are of dimensions $M_{K} \times\left(M_{k}-d_{1}\right)$ and $\left(M_{k}-d_{1}\right) \times d_{k}$, respectively; the columns of 
$\mathbf{V}_{k}$ should be a linear combination of the columns of $\mathbf{A}_{k}$. The desired signal is projected onto $\mathbf{U}_{1}$ at the PU's receiver, and interference signals from the secondary transmitters to PU's receiver should lie in the space spanned by the columns of $\left\{\mathbf{H}_{1 k} \mathbf{V}_{k}\right\}_{k=2}^{K}$. According to Equation (16), to avoid producing interference on the PU, the received interference at the PU should lie in the null space of $\mathbf{U}_{1}$. To achieve this, we aim to minimize the distance between the null space of $\mathbf{U}_{1}$ and the space spanned by the PU's received signal from the secondary links. To find the value of $\mathbf{A}_{k}$, we have the following proposition:

Proposition 2. Given $\mathbf{B}_{k}$ as the orthonormal bases of space $\mathcal{C}_{k}$, the $N_{1} \times M_{K}$ matrix $H_{1 k}, k \in \hat{\mathcal{K}}$ and the matrix $\mathbf{D}$ such that $\mathbf{D}=\mathbf{H}_{1 k} \mathbf{A}_{k}$. The minimum squared Euclidean distance between the subspace spanned by the columns of $\mathbf{D}$ and the space $\mathcal{C}_{k}$ can be obtained by $\mathbf{A}_{k}$ whose columns are equal to the eigenvectors corresponding to the $M_{k}-d_{1}$ minimum eigenvalues of $\mathbf{H}_{1 k}^{H} \mathbf{G}_{1} \mathbf{H}_{1 k}$, where $\mathbf{G}_{1}=\mathbf{I}_{N_{1}}-\mathbf{B}_{k} \mathbf{B}_{k}^{H}$.

Proof of Proposition 2. See [32], Lemma 1.

After finding the $\mathbf{A}_{k}$, to improve the performance for secondary links by a giving closed-form solution is equivalent to designing the transmitter to maximize the sum rate for a MIMO IFC: this does not exist in general. As an alternative, we propose a solution minimizing the interference leakage to the PU. The reverse direction interference covariance matrix at the receivers is $\mathbf{Q}_{k}=$ $P_{k} \mathbf{H}_{1 k} \mathbf{A}_{k} \mathbf{U}_{1} \mathbf{U}_{1}^{H} \mathbf{A}_{k}^{H} \mathbf{H}_{1 k}^{H}, k \in \hat{\mathcal{K}}$, then the columns of $\tilde{\mathbf{V}}_{k}$ are the $d_{k}$ least eigenvectors of reverse direction interference covariance matrix $\mathbf{Q}_{k}$ :

$$
\tilde{\mathbf{V}}_{k}=v_{\text {min }}^{d_{k}}\left(\mathbf{Q}_{k}\right)
$$

Furthermore, the optimal $\mathbf{V}^{*}$ that maximizes $\operatorname{SINR}_{k}$ is obtained in a closed form as [16]:

$$
\mathbf{U}_{k}^{*}=\left(\Phi_{k}\right)^{-1} \mathbf{H}_{k k} \mathbf{V}_{k} /\left\|\left(\Phi_{k}\right)^{-1} \mathbf{H}_{k k} \mathbf{V}_{k}\right\|
$$

where $\Phi_{k}=\sum_{j \neq k}^{K} \rho_{k} \mathbf{H}_{k j} \mathbf{V}_{j} \mathbf{V}_{j}^{H} \mathbf{H}_{k j}^{H}+\rho_{k} \sigma^{2} \mathbf{I}_{N}+\delta^{2} \mathbf{I}_{N}$. After finding the transceiver solution with given $\rho_{k}$ and $P_{k}$, we consider power allocation and power splitting ratio design with given $\mathbf{V}_{k}$ and $\mathbf{U}_{k}$.

$$
\begin{aligned}
& \max _{\left\{\rho_{k}, P_{k}\right\}} \sum_{k=2}^{K} \operatorname{SINR}_{k} \\
& \text { s.t. } \zeta_{k}\left(1-\rho_{k}\right)\left(\sum_{j=1}^{K} P_{j}\left\|\mathbf{H}_{k j} \mathbf{V}_{j}\right\|^{2}+\sigma_{k}^{2}\right) \geq e_{k}, \\
& \quad \sum_{k=2}^{K} P_{k}\left\|\mathbf{H}_{1 k}\right\|^{2} \geq Q_{\text {SUs-PU }}, \\
& \quad 0 \leq \rho_{k} \leq 1 \\
& \quad \sum_{k=2}^{K} P_{k}=P_{\text {total }}-P_{1}^{*} ; k \in \hat{\mathcal{K}} .
\end{aligned}
$$

It can be easily verified that the optimal $\rho_{k}^{*}$ satisfies the EH constraint of the problem in Equation (20) with equality. This conclusion can be proved by contradiction. Suppose the EH constraint of problem holds with inequality, for any power allocation schemes, we can increase the $\rho_{k}$ (i.e., decrease the $1-\rho_{k}$ ) until the EH constraints to equal, the $\mathrm{SINR}_{k}$ is increased as $\rho_{k}$ becomes bigger. Hence, the optimal $\rho_{k}^{*}$ for a given power allocation is $\rho_{k}^{*}=1-e_{k} / \zeta_{k}\left(\sum_{j=1}^{K} P_{j}\left\|\mathbf{H}_{k j} \mathbf{V}_{j}\right\|^{2}+\sigma_{k}^{2}\right)$. Given the $\rho_{k}$, the power allocation power problem can be expressed as: 


$$
\begin{gathered}
\max _{\left\{P_{k}\right\}} \sum_{k=2}^{K} \operatorname{SINR}_{k} \\
\text { s.t. } \zeta_{k}\left(1-\rho_{k}\right)\left(\sum_{j=1}^{K} P_{j}\left\|\mathbf{H}_{k j} \mathbf{V}_{j}\right\|^{2}+\sigma_{k}^{2}\right) \geq e_{k}, \\
\sum_{k=2}^{K} P_{k}\left\|\mathbf{H}_{1 k}\right\|^{2} \geq Q_{\mathrm{SUs}-\mathrm{PU}} \\
\sum_{k=2}^{K} P_{k}=P_{\text {total }}-P_{1}^{*} ; k \in \hat{\mathcal{K}} .
\end{gathered}
$$

This problem is nonconvex due to its objective function being nonconcave over $P_{k}$; by introducing variables $t_{k}$, we transform the the problem in Equation (21) into an equivalent convex problem as follows

$$
\begin{aligned}
& \max _{\left\{P_{k}, t_{k}\right\}} \sum_{k=2}^{K} \rho_{k} P_{k}\left|\mathbf{U}_{k}^{H} \mathbf{H}_{k k} \mathbf{V}_{k}\right|^{2} \\
& \text { s.t. } \sum_{j \neq k}^{K} \rho_{k} P_{j}\left|\mathbf{U}_{k}^{H} \mathbf{H}_{k j} \mathbf{V}_{j}\right|^{2}+t_{k}\left(\rho_{k} \sigma_{k}^{2}\left\|\mathbf{U}_{k}\right\|^{2}+\delta_{k}^{2}\left\|\mathbf{U}_{k}\right\|^{2}\right)=1, \\
& \quad \zeta_{k}\left(1-\rho_{k}\right)\left(\sum_{j=1}^{K} P_{j}\left\|\mathbf{H}_{k j} \mathbf{V}_{j}\right\|^{2}+\sigma_{k}^{2}\right) \geq t_{k} e_{k}, \\
& \sum_{k=2}^{K} P_{k}\left\|\mathbf{H}_{1 k}\right\|^{2} \geq t_{k} Q_{\text {sUs-PU }}, \\
& \quad t_{k}>0, \sum_{k=2}^{K} P_{k} / t_{k}=P_{\text {total }}-P_{1}^{*} ; k \in \hat{\mathcal{K}} .
\end{aligned}
$$

It can be easily verified that, given any feasible solution $\left\{P_{k}, t_{k}\right\}$ to the problem in Equation (22), the solution $\left\{P_{k} / t_{k}\right\}$ to the problem in Equation (21) attains the same objective value as that of the problem in Equation (22). Similarly, given any feasible solution $\left\{P_{k}\right\}$ to the problem in Equation (21), it can be shown that with solution $\left\{P_{k} /\left(\sum_{j \neq k}^{K} \rho_{k} P_{j}\left|\mathbf{U}_{k}^{H} \mathbf{H}_{k j} \mathbf{V}_{j}\right|^{2}+\rho_{k} \sigma_{k}^{2}\left\|\mathbf{U}_{k}\right\|^{2}+\delta_{k}^{2}\left\|\mathbf{U}_{k}\right\|^{2}\right)\right\}$, the problem achieves the same objective as that of the problem in Equation (21). Since the problem in Equation (22) is convex, this can be solved by CVX [31].

Our proposed sum rate maximization solution is summarized in Algorithm 1. Before executing Algorithm 1, we need to first check the feasibility of Equation (9) and therefore find initial $Q_{\text {SUs-PU }}$ and $\mathbf{U}_{1}$ for the first iteration. We initialize the $Q_{S U s-P U}=0$, and let the optimal receiver in transitional point-to point MIMO channel as the initial value of $\mathbf{U}_{1}$. We update channel realization until the problem in Equation (14) is solvable, and choose the corresponding $Q_{\mathrm{SUs}-\mathrm{PU}}=0$ and $\mathbf{U}_{1}$ as the initial solution for executing the Algorithm 1. 


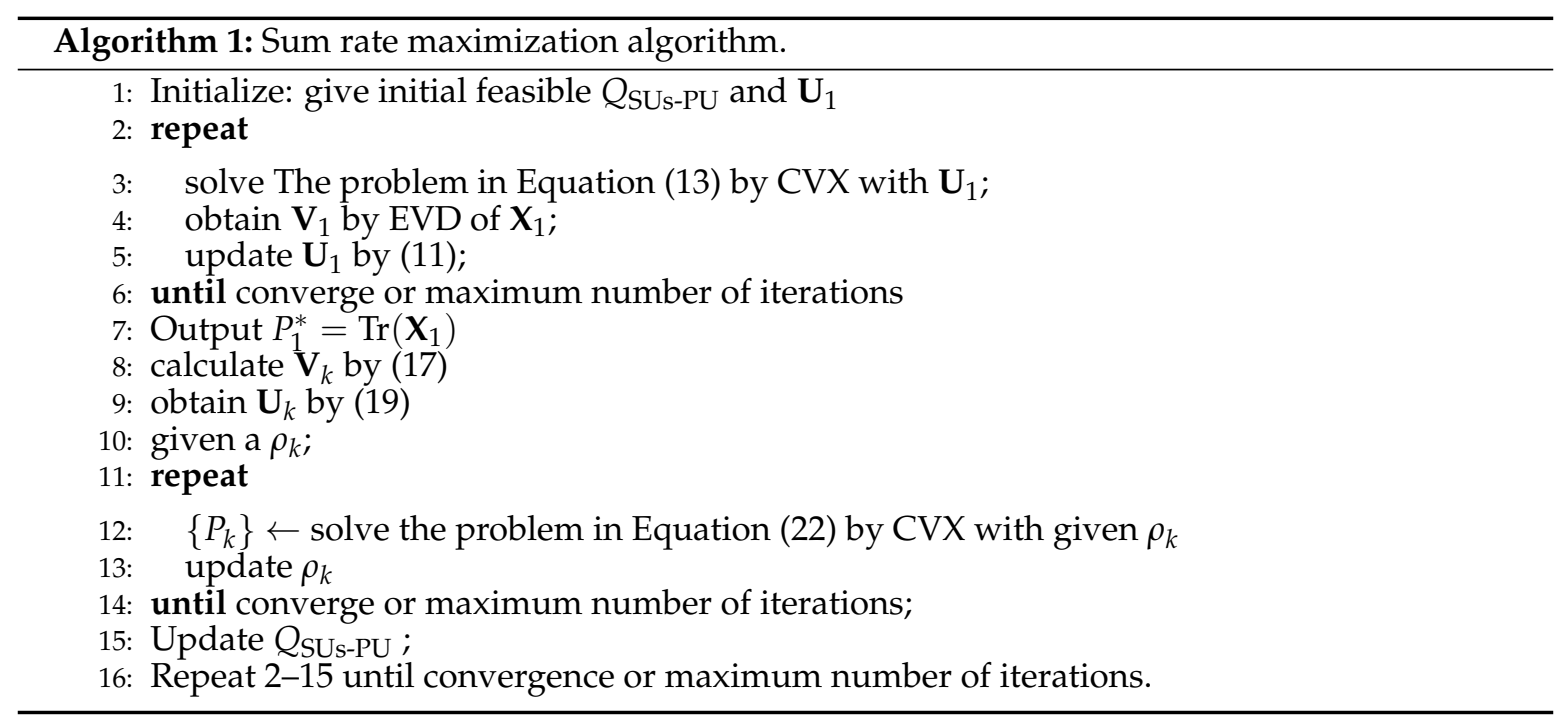

\subsection{Maximize Minimum SINR Solution for SUs}

Next, to avoid undesired SINR compromise by the sum rate maximization solution, we propose another solution to maximize the minimum SINR of SUs; the interference from the SUs to the PU need be aligned into a same subspace, the SUs' precoder should be the same as that in the Sum-Rate maximization solution, hence we only focus on the power allocation and power splitting ratio design in this problem, which is formulated as:

$$
\begin{aligned}
& \max _{\left\{\rho_{k}, P_{k}\right\}} \min _{k} \operatorname{SINR}_{k} \\
& \text { s.t. } \zeta_{k}\left(1-\rho_{k}\right)\left(\sum_{j=1}^{K} P_{j}\left\|\mathbf{H}_{k j} \mathbf{V}_{j}\right\|^{2}+\sigma_{k}^{2}\right) \geq e_{k}, \\
& \quad 0 \leq \rho_{k} \leq 1, \\
& \quad 0 \leq P_{k} \leq P_{k}^{\max }, \\
& \sum_{k=2}^{K} P_{k}=P_{\text {total }}-P_{1}^{*} ; k \in \hat{\mathcal{K}} .
\end{aligned}
$$

It can easily be checked that problem is feasible if, and only if, $\zeta_{k} \sum_{j=2}^{K} P_{j}^{\max }\left\|\mathbf{H}_{j k} \mathbf{V}_{j}\right\|^{2} \geq e_{k}$, $k=2, \ldots, K$ for given $e_{k}$ and $P_{k}^{\max }$. We assume that is always feasible; the problem is nonconvex since the transmit power $P_{k}$ and the PS ratio $\rho_{k}$ are coupled with each other in both the objective function and the EH constraints. To make the problem tractable, we introduce a real-valued slack variable $\alpha$ and then reformulate the problem as:

$$
\begin{aligned}
& \max _{P_{k}, \rho_{k}, \alpha} \alpha \\
& \text { s.t. } \alpha \geq 0, \alpha-\operatorname{SINR}_{k} \leq 0, \\
& \\
& e_{k}-\zeta_{k}\left(1-\rho_{k}\right)\left(\sum_{j=1}^{K} P_{j}\left\|\mathbf{H}_{j k} \mathbf{V}_{j}\right\|^{2}+\sigma_{k}^{2}\right) \leq 0, \\
& 0 \leq \rho_{k} \leq 1, \\
& 0 \leq P_{k} \leq P_{k}^{\max }, \\
& \sum_{k=2}^{K} P_{k}=P_{\text {total }}-P_{1}^{*} ; k \in \hat{\mathcal{K}}, .
\end{aligned}
$$


The problem in Equation (24) is convex for any given $\alpha$, thus searching $\alpha$ over a specific and solving the problem at each step with the associated $\alpha$ can obtain the optimal solution. Here, we give some properties of the problem in Equation (24). If $P_{k}^{\max } \leq P_{\text {total }}-P_{1}^{*}$ for $k \in \hat{\mathcal{K}}$, then we have the following propositions:

Proposition 3. If the SINR of link $k$ is strictly less than that of all other links, i.e., $\operatorname{SINR}_{k}^{*}<\operatorname{SINR}_{j}^{*}, \forall k \neq j$, then $P_{k}^{*}=P_{k}^{\max }$.

Proof of Proposition 3. Suppose this not true; we can increase transmit power within its maximum power constraint, and decrease the $j$ transmit power until both the SINR values are equal. This shows that the Max-Min SINR can always be increased until the Min-Min SINR reaches its maximum power.

Proposition 4. If the SINR of link $k$ is strictly less than that of all other links, i.e., $\operatorname{SINR}_{k}^{*}<\operatorname{SINR}_{j}^{*}, \forall k \neq j$, the equality of the $E H$ constraint holds at the receiver $k$, i.e., $Q_{k}=e_{k}$, while $Q_{j} \geq e_{j}$ at receiver $j$.

Proof of Proposition 4. Suppose that this is not true. That is, if $\operatorname{SINR}_{k}<\mathrm{SINR}_{j}$, the harvested power at receiver $k$ is $Q_{k}>e_{k}$. In this case, we can decrease $\rho_{k}$ to increase $\operatorname{SINR}_{k}$ until $Q_{k}=e_{k}$, which in turn increases the Max-Min SINR value.

\subsection{Solution Discussion}

In this section, we discuss some importance issues to implement the proposed centralized solution. Generally speaking, all CSI should be globally available for centralized solutions [14], due to PU's priority setting in our system model, only all SUs' CSI should be known at the transceiver of all users to operate the algorithm. Thus, our proposed solution to reduce the CSI overhead of PU is consistent with the principle of CR. Nevertheless, to implement the CSI exchange among users, a dedicated central processing unit should be deployed to collect CSI and then send that to corresponding transmitters/receivers [14]. In fact, it is advisable to apply our proposed solution for slow fading channels.

Besides the CSI overhead, perfect CSI in transmitter and receiver are assumed. In practice, acquiring perfect CSI is a challenge due to many factors, such as channel estimation error, feedback delay, and the limited onboard energy. Note that the IA solution proposed in this paper has the assumption that the CSI is perfect; once the CSI changes, the solution of transceivers and power allocation may no long match reality, thus imperfect CSI would lead to performance degradation. To that end, various robust transceiver designs have been studied for different networks (e.g., [13,25,27]). Hence, the imperfect CSI situation is beyond the consideration of this paper.

Another concern is the energy harvesting model issue. In this paper, we assume the energy conversion efficiency is a constant dependent of transmit power. The $\mathrm{EH}$ model being considered in this paper is a liner model, which has the benefit of being analytically easily tractable. Indeed, the energy conversion efficiency is not only a function of input signal shape and power, but also a function of the transmit signal (beamforming, waveform, modulation, and power allocation) and the wireless channel state [33]. There has been an increasing interest in the SWIPT literature to study the nonlinear model, which describes the nonlinear characteristics of practical RF EH circuits [33-35]. Note that in a nonlinear EH model, the CSCG inputs cannot achieve the Pareto optimal solution of the R-E region [34]. Studies on input distribution, modulation, and waveform designs are needed for nonlinear EH networks [35]. Moreover, research is in its infancy when it comes to multi-user SWIPT design for the nonlinear models; to our knowledge, the R-E tradeoff of multi-user MIMO IFC remains largely unknown. Thus, we consider a liner EH model in this paper, and the main idea applying IA to protect a PU's priority and manage the interference can be extended to the nonlinear case. It important to point out that the power allocation and beamforming radically changes once we change the energy harvester model and adopt more realistic nonlinear models of the energy harvester. The results present 
in this paper give a lower bound the for performance in practical nonlinear cases. It is of interest to re-think the power allocation and transceiver design for the nonlinear case in the future.

Finally, we discuss the computational complexity of Algorithm 1. CVX software [31] was applied to solve the problem in Equation (14). The complexity of solving the problem in Equation (14) is $\mathcal{O}\left(\sqrt{M}\left(M^{2}+M^{3}\right)\right)$; the complexity of solving the problem in Equation (15) arises from the EVD operation to compute the $\mathbf{V}_{k}$ and $\mathbf{U}_{k}, k \in \hat{\mathcal{K}}$. Suppose the number of iteration to solve Equation (15) is $T_{\max }$, the number of iterations to operate Algorithm 1 is $X_{\max }$, if $M \geq N$, the complexity of Algorithm 1 is $\mathcal{O}\left(X_{\max } \sqrt{M}\left(M^{2}+M^{3}\right)+X_{\max } T_{\max } K M^{3}\right)$, else $\mathcal{O}\left(X_{\max } \sqrt{M}\left(M^{2}+M^{3}\right)+X_{\max } T_{\max } K N^{3}\right)$.

\section{Simulation Results}

In this section, we provide some numerical simulation examples to validate the performance of the proposed approaches. We assume there is one PU with three SUs and all users have the same set of parameters; each user is equipped with $M_{k}=M, N_{k}=N$ antennas. The signal attenuation from transmitter to receiver is set as $40 \mathrm{~dB}$ for the direct channels and as $40+10 \log _{10} \varepsilon \mathrm{dB}$ for the cross-link channels [15], where $\varepsilon$ is set as relative cross-link gain. Specifically, the variance of channel coefficients is $\omega_{k k}=10^{-4}$ and $\omega_{k j}=10^{-4} / \varepsilon$ for all $k, j \in \mathcal{K}, k \neq j$. Moreover, $\zeta=0.5, \sigma^{2}=-70 \mathrm{dBm}$, and $\delta^{2}=-50 \mathrm{dBm}$. The distance between transmitter and receiver is set as $3 \mathrm{~m}$, and the frequency of the carrier wave is assumed to be $900 \mathrm{MHz}$. The EH thresholds are set as being equal for all SUs, $e_{k}=e$. We take the IA through all the users with the average power allocation (IA-APA) as benchmark. The total transmission power of the proposed solutions is set to be equal to that of full IA under the same constraints.

First, we study the transmission power required at the PU versus the SINR target $\gamma_{1}$ for the proposed solutions, with fixed parameters $M_{k}=N_{k}=4$; all users' EH threshold is $-10 \mathrm{dBm}$. Total transmit power is set to be equal to that of IA-APA solution under the same conditions. Figure 2 shows the performance comparison of the proposed solutions in terms of the minimum transmit power, and it can be seen that the sum rate maximization solution has the best performance. It is observed that, as the SINR $\gamma_{1}$ is increased from $0 \mathrm{~dB}$ to $40 \mathrm{~dB}$, more transmission power is needed, and the performance gap between the full IA solution and Max-Min solution gets closer, and Figure 2 also shows that the performance of all solutions is not sensitive to the increasing SINR targets at low to medium SINR; this is because the EH target is quite demanding.

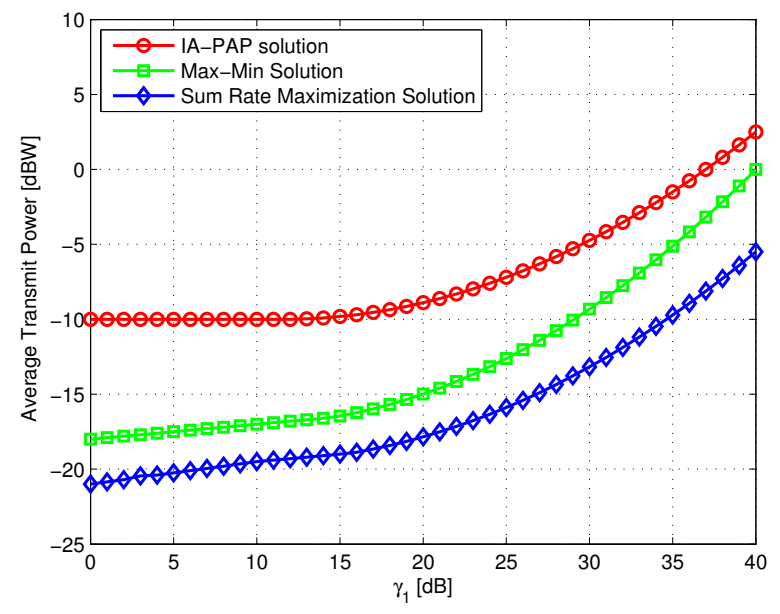

Figure 2. PU's minimum transmit power versus SINR targets.

Next, we show in Figure 3 the minimum transmission power of the proposed solutions over $e_{1}$ with fixed $\gamma_{1}=10 \mathrm{~dB}, M=N=4$ and $\varepsilon=1$. In Figure 3 the optimal solution achieves the minimum PU transmission power. Furthermore, the proposed solutions achieve notably smaller 
transmission power than that of the full IA solution because the PU designs its precoder selfishly in the proposed solutions.

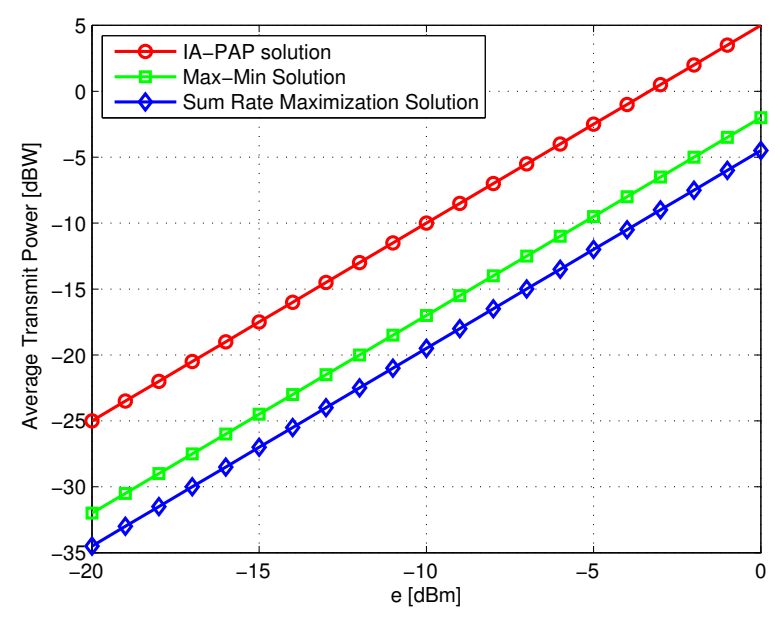

Figure 3. PU's minimum transmit power versus EH targets.

Figure 4 plots the minimum transmit power versus the relative cross-link gain $\varepsilon$, with fixed $M_{k}=N_{k}=4, e_{1}=-10 \mathrm{dBm}$ and $\gamma_{1}=10 \mathrm{~dB}$. As cross-link channels become weaker, i.e., the $\varepsilon$ increases, the interference from the SUs to the PU becomes smaller, so the PU harvests less energy from SUs, so more transmit power is required to obtain the same SINR and EH target.

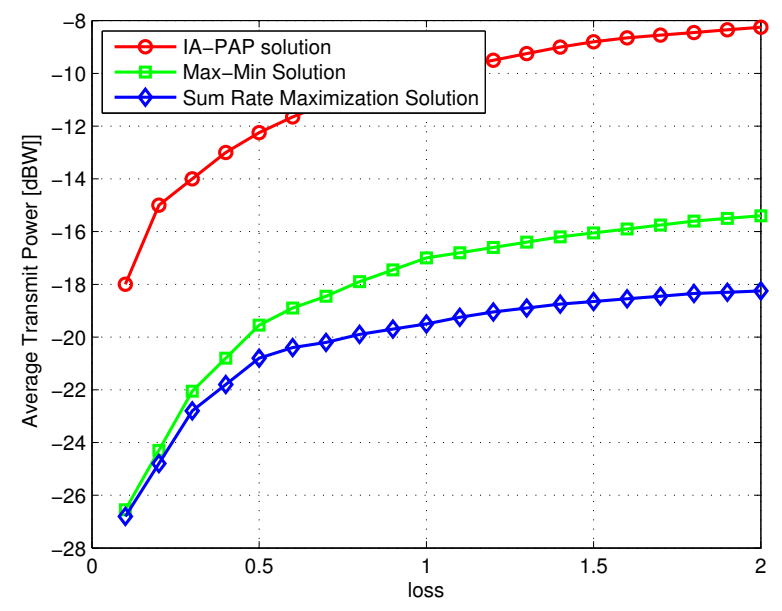

Figure 4. PU's minimum transmit power versus cross-link gains.

We show in Figure 5 the average SINR of SUs achieved by the proposed solutions, with $M_{k}=N_{k}=4, \gamma_{1}=10 \mathrm{~dB}, e=-10 \mathrm{dBm}$ and $\varepsilon=1$. It is found that the sum rate maximization solution achieves the highest SINR and the Max-Min solution achieves the lowest SINR, because the Max-Min solution cares about users' fairness among the SUs, transmit power mainly being allocated to improve the performance of the SUs who achieve the lowest SINR. We plot the Max and Min SINR of SUs achieved by each solution in Figure 6, which shows the optimal solution achieves the best Max SINR, the lowest Min SINR being obtained by the sum rate maximization solution. Moreover, the Max-Min solution guarantees the users' fairness, whereas the maximum minimum SINR is achieved by the Max-Min solution. 


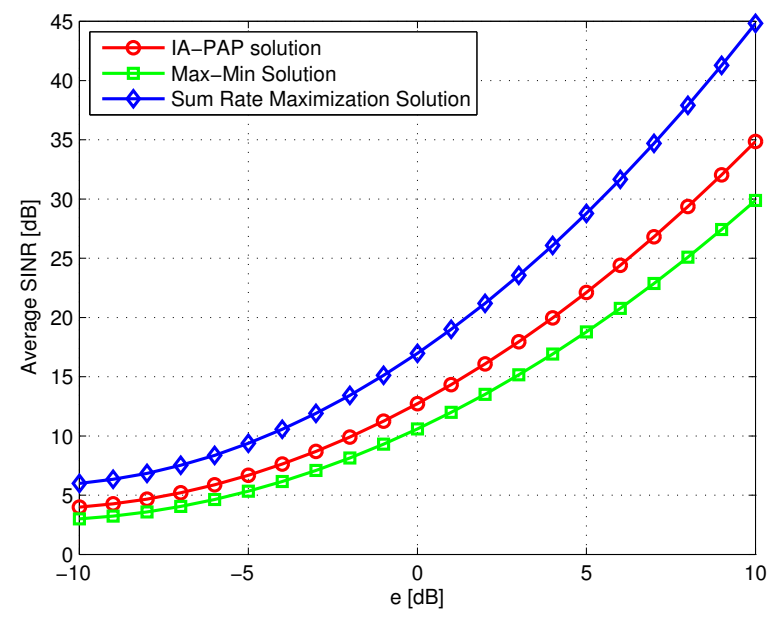

Figure 5. SUs' average SINR versus EH targets.

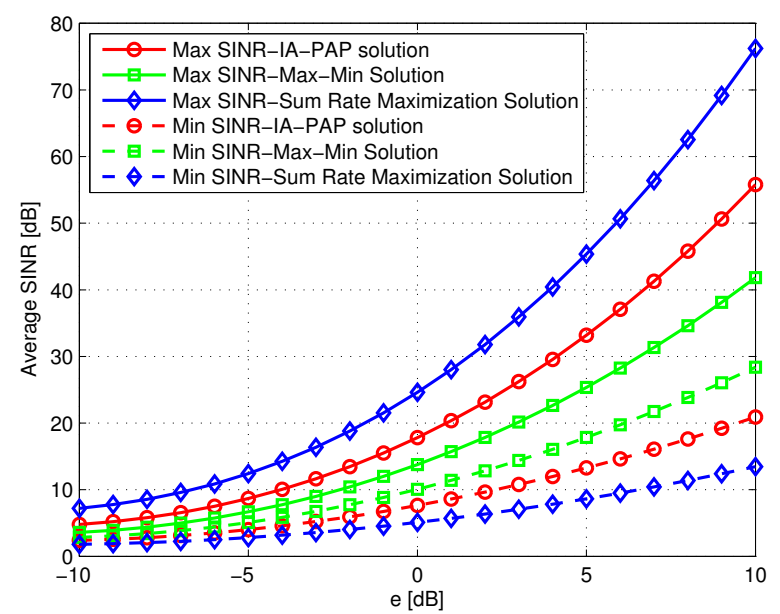

Figure 6. SUs' Max SINR and Min SINR versus EH targets.

Finally, we provide numerical results to verify the fast convergence speed of the proposed solutions. The average achievable SINR of both sum rate maximization and Max-Min solutions is depicted as a function of iterations, with $M_{k}=N_{k}=4, \gamma_{1}=10 \mathrm{~dB}, e=-10 \mathrm{dBm}$ and $\varepsilon=1$. In Figure 7 the proposed approaches converges in a few iterations. Our solutions indeed converge by iteration process.

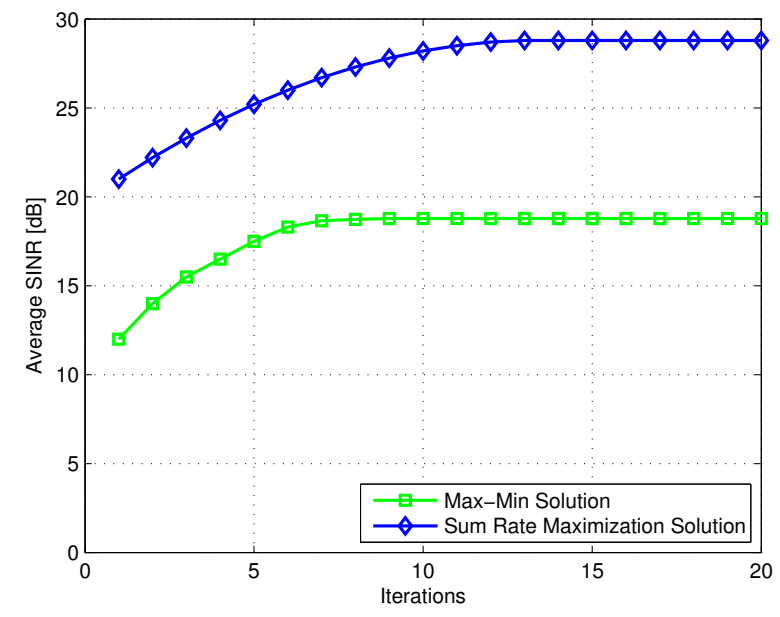

Figure 7. SU's average SINR versus iterations. 


\section{Conclusions}

This paper studies the transceiver design and power allocation for SWIPT over MIMO CR networks. We solved the nonconvex problem by alternating optimization in which the problem was decoupled into several optimization problems. By reformulation, the transmission power at the PU is minimized by SDR, while satisfying SINR and harvested energy constraints of the PU. The transceiver and power allocation are iteratively optimized by convex optimization for sum rate maximization and Max-Min approaches. Simulation shows different influences of SINR thresholds, EH thresholds and the cross-link interference on the performance of different solutions. The useful insights provided in this paper may help future investigations in the following research directions:

- Robust design: This paper considers a perfect CSI among all users, thus extending the results in our work to the more general imperfect CSI case is an interesting topic.

- Massive MIMO: Massive MIMO is a promising technology in the 5G communication network, but more effort is needed to design the transceiver for a Massive MIMO case.

- Nonlinear energy harvesting model: In practice, the energy harvesting model is a nonlinearity model; in the near future, we will pay attention to transmission strategies design for nonlinear energy harvesting model.

Author Contributions: writing-original draft preparation, F.W.; supervision, L.X.; funding acquisition, L.X. and D.Y.; writing—review and editing, L.C.; and project administration, X.L.

Funding: This research was funded by the National Natural Science Foundation of China under grant numbers 61703197, 61561032 and 61461029.

Conflicts of Interest: The authors declare no conflict of interest.

\section{Appendix A. Proof of the Proposition 1}

Since the problem is convex and satisfies the Slater's condition, it has zero duality gap. The Lagrangian of Equation (14) can be expressed as

$$
\begin{aligned}
& L\left(\mathbf{X}_{1}, \rho_{1}, \lambda_{1}, \mu_{1}\right) \triangleq \operatorname{Tr}\left(\mathbf{X}_{1}\right) \\
& -\lambda_{1}\left(\frac{1}{\gamma_{1}} \mathbf{U}_{1}^{H} \mathbf{H}_{11} \mathbf{X}_{1} \mathbf{H}_{11}^{H} \mathbf{U}_{1}-\sigma_{1}^{2}-\frac{\delta_{1}^{2}}{\rho_{1}}\right) \\
& -\mu_{1}\left(Q_{\text {SUs-PU }}-\left\|\mathbf{H}_{11} \mathbf{X}_{1} \mathbf{H}_{11}^{H}\right\|-\sigma_{1}^{2}-\frac{e_{1}}{\zeta_{1}\left(1-\rho_{1}\right)}\right)
\end{aligned}
$$

where $\lambda_{1}$ and $\mu_{1}$ denote the dual variables associated with the SINR constraint and harvested energy constraint of the problem in Equation (14), respectively. The dual function of the problem in Equation (14) is expressed as:

$$
\mathrm{g}\left(\lambda_{1}, \mu_{1}\right)=\min _{\mathbf{X}_{1} \succeq 0,0<\rho_{1}<1} L\left(\mathbf{X}_{1}, \rho_{1}, \lambda_{1}, \mu_{1}\right)
$$

This can be rewritten as

$$
\begin{aligned}
& \mathrm{g}\left(\lambda_{1}, \mu_{1}\right)=\min _{\mathbf{X}_{1} \succeq 0,0<\rho_{1}<1} \operatorname{Tr}\left(\Psi \mathbf{X}_{1}\right)+\left(\lambda_{1}+\mu_{1}\right) \sigma_{1}^{2} \\
& +\left[\frac{\lambda_{1} \delta_{1}^{2}}{\rho_{1}}+\mu_{1}\left(\frac{e_{1}^{2}}{\zeta_{1}\left(1-\rho_{1}\right)}-Q_{\text {SUs-PU }}\right)\right]
\end{aligned}
$$

where

$$
\Psi=\mathbf{I}_{M}-\frac{\lambda_{1}}{\gamma_{1}} \mathbf{U}_{1}^{H} \mathbf{H}_{11} \mathbf{H}_{11}^{H} \mathbf{U}_{1}-\mu_{1} \mathbf{H}_{11} \mathbf{H}_{11}^{H}
$$

Since the problem can be solved equivalently by solving the problem in Equation (A2), i.e., first minimizing the Lagrangian to obtain the dual function with fixed $\lambda_{1}>0$ and $\mu_{1}>0$, and then 
searching the optimal dual solutions $\lambda_{1}^{*}$ and $\mu_{1}^{*}$ to maximize the dual function, the $\mathbf{X}_{1}^{*}$ and $\rho_{1}^{*}$ that maximize the Lagrangian to obtain $\mathrm{g}\left(\lambda_{1}^{*}, \mu 1^{*}\right)$ are the optimal solution of Equation (14).

Let $\lambda_{1}^{*}$ and $\mu_{1}^{*}$ denote the optimal dual solution, we define

$$
\Psi^{*}=\mathbf{I}_{M}-\frac{\lambda_{1}^{*}}{\gamma_{1}} \mathbf{U}_{1}^{H} \mathbf{H}_{11} \mathbf{H}_{11}^{H} \mathbf{U}_{1}-\mu_{1}^{*} \mathbf{H}_{11} \mathbf{H}_{11}^{H}
$$

It is observed from Equation (A3) that $\mathbf{X}_{1}$ and $\rho_{1}$ are decoupled with each other, hence $\mathbf{X}_{1}^{*}$ must be a solution to the following problem:

$$
\min _{\mathbf{X}_{1} \succeq 0} \operatorname{Tr}\left(\Psi^{*} \mathbf{X}_{1}\right)
$$

To guarantee a bounded dual optimal value, we must have

$$
\Psi^{*} \succeq 0
$$

As a result, the optimal value of problem is zero, i.e., $\operatorname{Tr}\left(\Psi^{*} \mathbf{X}_{1}\right)=0$; together with $\Psi^{*} \succeq 0$ and $\mathbf{X}_{1} \succeq 0$, it follows that:

$$
\Psi^{*} \mathbf{X}_{1}^{*}=0
$$

Note that $\mathbf{I}_{M}-\frac{\lambda_{1}^{*}}{\gamma_{1}} \mathbf{U}_{1}^{H} \mathbf{H}_{11} \mathbf{H}_{11}^{H} \mathbf{U}_{1}$ is Hermitian and Positive Definite, hence $\operatorname{Rank}\left(\mathbf{I}_{M}-\right.$ $\left.\frac{\lambda_{1}^{*}}{\gamma_{1}} \mathbf{U}_{1}^{H} \mathbf{H}_{11} \mathbf{H}_{11}^{H} \mathbf{U}_{1}\right)=M$, and it follows that $\operatorname{Rank}\left(\Psi^{*}\right) \geq M-1$. If $\operatorname{Rank}\left(\Psi^{*}\right)=M$, from Equation (A8), we have $\mathbf{X}_{1}^{*}=0$, i.e., the transmit power of PU is zero, which cannot be the optimal solution to the problem. As a result, $\operatorname{Rank}\left(\Psi^{*}\right)=M-1$. From Equation (A8), we have $\operatorname{Rank}\left(\mathbf{X}_{1}^{*}\right)=1$ so the first part of proposition is thus proved.

Next, we consider the second part of Proposition 1.The optimal $\rho_{1}^{*}$ can be found by solving the following problem

$$
\min _{0<\rho_{1}<1} \frac{\lambda_{1}^{*} \delta_{1}^{2}}{\rho_{1}}+\mu_{1}^{*}\left(\frac{e_{1}^{2}}{\zeta_{1}\left(1-\rho_{1}\right)}-Q_{\text {SUs-PU }}\right)
$$

It is observed from Equation (A9) that, if $\lambda_{1}^{*}=0$ and $\mu_{1}^{*}>0$, the optimal solution is $\rho_{1}^{*} \rightarrow 1$. Besides, if $\lambda_{1}^{*}>0$ and $\mu_{1}^{*}=0$, the optimal solution is $\rho_{1}^{*} \rightarrow 0$. However, for given $\gamma_{1}>0$ and $e_{1}>0$, these two cases cannot happen. Furthermore, if $\lambda_{1}^{*}=0$ and $\mu_{1}^{*}=0$, we have $\Psi^{*}=\mathbf{I}_{M}$, which turns to $\mathbf{X}_{1}^{*}=0$, hence $\lambda_{1}^{*}=0$ and $\mu_{1}^{*}=0$ cannot be true. In conclusion, the optimal dual variables should satisfy $\lambda_{1}^{*}>0$ and $\mu_{1}^{*}>0$, according to the complementary slackness [36]; the second part of Proposition 1 is thus proved. Combing the proofs of both parts, the Proposition 1 is proved.

\section{References}

1. Valtchev, S.; Borge, B.V.; Brandisky, K.; Klaassens, J.B. Efficient Resonant Inductive Coupling Energy Transfer Using New Magnetic and Design Criteria. In Proceedings of the 2005 IEEE 36th Power Electronics Specialists Conference, Recife, Brazil, 16 June 2005; pp. 1293-1298.

2. Liu, H. Maximizing Efficiency of Wireless Power Transfer with Resonant Inductive Coupling. 2011. Available online: http://hxhl95.github.io/media/ib_ee.pdf (accessed on 16 October 2018).

3. Kurs, A.; Karalis, A.; Moffatt, R.; Joannopoulos, J.D.; Fisher, P.; Soljačić, M. Wireless power transfer via strongly coupled magnetic resonances. Science 2007, 317, 83-86. [CrossRef] [PubMed]

4. Jonah, O.; Georgakopoulos, S.V. Wireless Power Transfer in Concrete via Strongly Coupled Magnetic Resonance. IEEE Trans. Antennas Propag. 2013, 61, 1378-1384. [CrossRef]

5. Varshney, L.R. Transporting information and energy simultaneously. In Proceedings of the 2008 IEEE International Symposium on Information Theory, Toronto, ON, Canada, 6-11 July 2008; pp. 1612-1616.

6. Lu, X.; Wang, P.; Niyato, D.; Kim, D.I.; Han, Z. Wireless Networks with RF Energy Harvesting: A Contemporary Survey. IEEE Commun. Surv. Tutor. 2015, 17, 757-789. [CrossRef] 
7. Yang, D.; Wu, Q.; Zeng, Y.; Zhang, R. Energy Tradeoff in Ground-to-UAV Communication via Trajectory Design. IEEE Trans. Veh. Technol. 2018, 67, 6721-6726.

8. Zhang, R.; Ho, C.K. MIMO Broadcasting for Simultaneous Wireless Information and Power Transfer. IEEE Trans. Wirel. Commun. 2013, 12, 1989-2001. [CrossRef]

9. Liu, L.; Zhang, R.; Chua, K.C. Wireless Information Transfer with Opportunistic Energy Harvesting. IEEE Trans. Wirel. Commun. 2013, 12, 288-300. [CrossRef]

10. Ng, D.W.K.; Lo, E.S.; Schober, R. Wireless Information and Power Transfer: Energy Efficiency Optimization in OFDMA Systems. IEEE Trans. Wirel. Commun. 2013, 12, 6352-6370. [CrossRef]

11. Wen, Z.; Liu, X.; Zheng, S.; Guo, W. Joint Source and Relay Design for MIMO Two-Way Relay Networks with SWIPT. IEEE Trans. Veh. Technol. 2018, 67, 822-826. [CrossRef]

12. Shi, Q.; Liu, L.; Xu, W.; Zhang, R. Joint Transmit Beamforming and Receive Power Splitting for MISO SWIPT Systems. IEEE Trans. Wirel. Commun. 2014, 13, 3269-3280. [CrossRef]

13. Wang, F.; Peng, T.; Huang, Y. Decentralized Robust Transceiver Designs for MISO SWIPT Interference Channel. IEEE Access 2018, 6, 4537-4546. [CrossRef]

14. Zong, Z.; Feng, H.; Yu, F.R.; Zhao, N.; Yang, T.; Hu, B. Optimal Transceiver Design for SWIPT in K-User MIMO Interference Channels. IEEE Trans. Wirel. Commun. 2016, 15, 430-445. [CrossRef]

15. Zhao, M.; Cai, Y.; Shi, Q.; Hong, M.; Champagne, B. Joint Transceiver Designs for Full-Duplex K-Pair MIMO Interference Channel with SWIPT. IEEE Trans. Commun. 2017, 65, 890-905. [CrossRef]

16. Gomadam, K.; Cadambe, V.; Jafar, S. A distributed numerical approach to interference alignment and applications to wireless interference networks. IEEE Trans. Inf. Theory 2011, 57, 3309-3322. [CrossRef]

17. Zhao, N.; Yu, F.R.; Leung, V.C.M. Wireless energy harvesting in interference alignment networks. IEEE Commun. Mag. 2015, 53, 72-78. [CrossRef]

18. Li, X.; Sun, Y.; Yu, F.R.; Zhao, N. Antenna selection and power splitting for simultaneous wireless information and power transfer in interference alignment networks. In Proceedings of the 2014 IEEE Global Communications Conference, Austin, TX, USA, 8-12 December 2014; pp. 2667-2672.

19. Guo, J.; Zhao, N.; Yu, F.R.; Liu, X.; Leung, V.C.M. Exploiting Adversarial Jamming Signals for Energy Harvesting in Interference Networks. IEEE Trans. Wirel. Commun. 2017, 16, 1267-1280. [CrossRef]

20. Lunden, J.; Koivunen, V.; Huttunen, A.; Poor, H.V. Collaborative Cyclostationary Spectrum Sensing for Cognitive Radio Systems. IEEE Trans. Signal Process. 2009, 57, 4182-4195. [CrossRef]

21. Lee, W.; Cho, D. Enhanced Spectrum Sensing Scheme in Cognitive Radio Systems with MIMO Antennae. IEEE Trans. Veh. Technol. 2011, 60, 1072-1085. [CrossRef]

22. Rossi, P.S.; Ciuonzo, D.; Romano, G. Orthogonality and Cooperation in Collaborative Spectrum Sensing through MIMO Decision Fusion. IEEE Trans. Wirel. Commun. 2013, 12, 5826-5836. [CrossRef]

23. Mohammad, F.R.; Ciuonzo, D.; Mohammed, Z.A.K. Mean-Based Blind Hard Decision Fusion Rules. IEEE Signal Process. Lett. 2018, 25, 630-634. [CrossRef]

24. Yang, Z.; Ding, Z.; Fan, P.; Karagiannidis, G.K. Outage Performance of Cognitive Relay Networks with Wireless Information and Power Transfer. IEEE Trans. Veh. Technol. 2016, 65, 3828-3833. [CrossRef]

25. Zhou, F.; Li, Z.; Cheng, J.; Li, Q.; Si, J. Robust AN-Aided Beamforming and Power Splitting Design for Secure MISO Cognitive Radio with SWIPT. IEEE Trans. Wirel. Commun. 2017, 16, 2450-2464. [CrossRef]

26. Tuan, P.V.; Koo, I. Optimal Multiuser MISO Beamforming for Power-Splitting SWIPT Cognitive Radio Networks. IEEE Access 2017, 5, 14141-14153. [CrossRef]

27. Xu, C.; Zhang, Q.; Li, Q.; Tan, Y.; Qin, J. Robust Transceiver Design for Wireless Information and Power Transmission in Underlay MIMO Cognitive Radio Networks. IEEE Commun. Lett. 2014, 18, 1665-1668. [CrossRef]

28. Fang, B.; Qian, Z.; Zhong, W.; Shao, W. AN-Aided Secrecy Precoding for SWIPT in Cognitive MIMO Broadcast Channels. IEEE Commun. Lett. 2015, 19, 1632-1635. [CrossRef]

29. Yuan, Y.; Ding, Z. Outage Constrained Secrecy Rate Maximization Design with SWIPT in MIMO-CR Systems. IEEE Trans. Veh. Technol. 2018, 67, 5475-5480. [CrossRef]

30. Wu, F.; Xiao, L.; Yang, D.; Cuthbert, L.; Liu, X. Transceiver Designs for Interference Alignment Based Cognitive Radio Networks with Energy Harvesting. Wirel. Pers. Commun. 2018, 98, 1895-1911. [CrossRef]

31. Grant, M.; Boyd, S. CVX: Matlab Software for Disciplined Convex Programming, Version 2.0 Beta; CVX Research, Inc.: Austin, TX, USA, 2013. 
32. Rezaei, F.; Tadaion, A. Sum-Rate Improvement in Cognitive Radio Through Interference Alignment. IEEE Trans. Veh. Technol. 2016, 65, 145-154. [CrossRef]

33. Boshkovska, E.; Ng, D.W.K.; Zlatanov, N.; Schober, R. Practical Non-Linear Energy Harvesting Model and Resource Allocation for SWIPT Systems. IEEE Commun. Lett. 2015, 19, 2082-2085. [CrossRef]

34. Clerckx, B.; Bayguzina, E. Waveform Design for Wireless Power Transfer. IEEE Trans. Signal Process. 2016, 64, 6313-6328. [CrossRef]

35. Zeng, Y.; Clerckx, B.; Zhang, R. Communications and Signals Design for Wireless Power Transmission. IEEE Trans. Commun. 2017, 65, 2264-2290. [CrossRef]

36. Boyd, S.; Vandenberghe, L. Convex Optimization; Cambridge University Press: Cambridge, UK, 2004.

(C) 2018 by the authors. Licensee MDPI, Basel, Switzerland. This article is an open access article distributed under the terms and conditions of the Creative Commons Attribution (CC BY) license (http://creativecommons.org/licenses/by/4.0/). 